\title{
Jovian, Solar, and other Possible Sources of Radiation Belt Particles
}

\author{
D.N. Baker ${ }^{1}$, S.G. Kanekal ${ }^{2}$, M.D. Looper ${ }^{3}$, J.B. Blake ${ }^{3}$, and R.A. Mewaldt ${ }^{4}$
}

\begin{abstract}
It is well known that electrons, protons, and heavier ions can be accelerated to high energies $(\gtrsim 1 \mathrm{MeV})$ throughout the solar system by a variety of mechanisms. We review several of the sources of energetic ions and electrons that can produce enhanced fluxes of particles near the Earth's orbit. Solar energetic particles and particles accelerated at interplanetary shock waves are considered. We also review the properties and potential terrestrial influence of Jovian electrons. Recent measurements from the SAMPEX spacecraft in low-Earth orbit are examined to look for extraterrestrial sources of electrons and ions. We find clear evidence of both solar and Jovian electrons at high latitudes and at high altitudes around the Earth, but the durably trapped outer zone electron population seems best and most completely explained by an internal acceleration mechanism.
\end{abstract}

\section{INTRODUCTION}

It is widely recognized that the plasma populating the Earth's magnetosphere may originate both from the ionosphere (e.g., Gloeckler and Hamilton [1987]) and from the solar wind (e.g., Cowley [1980]). High-energy particles within the Earth's radiation belts can, in principle, be accelerated in situ in the magnetosphere [Schulz and Lanzerotti, 1974] or they can originate from sources beyond the Earth's immediate influence [Scholer, 1979]. Notable possible source regions include solar flare sites in the sun's atmosphere [Mason et al., 1994], interplanetary shock waves driven by solar disturbances [Fisk, 1971], and corotating interplanetary stream interaction regions [Gloeckler, 1984]. It is also known that Jupiter's magnetosphere is a copious source of relativistic electrons which fills the inner solar system (e.g., Teegarden et al. [1974]) and these electrons have been suggested as a possible source of high-energy electrons in Earth's outer radiation belt [Baker et al., 1979].

Our purpose in this paper is to examine sources of radiation belt particles external to the Earth's magnetosphere. We will particularly focus on sources that may be responsible for

\footnotetext{
${ }^{1}$ LASP, University of Colorado, Boulder, Colorado

${ }^{2}$ NASA/Goddard Space Flight Center, Greenbelt, Maryland

${ }^{3}$ Aerospace Corp., Los Angeles, California

${ }^{4}$ California Inst. of Tech., Pasadena, California
}

Radiation Belts: Models and Standards

Geophysical Monograph 97

Copyright 1996 by the American Geophysical Union the highly dynamic and (often) regularly variable relativistic electron population in Earth's outer radiation zone.

\section{ENERGETIC ION SOURCES IN THE HELIOSPHERE}

Figure 1 shows in a schematic way the energetic ion populations of the solar system. As is evident, the region near 1 astronomical unit (AU) is awash with energetic ions which have been accelerated in a wide variety of locations. Among the particle populations of interest are solar energetic particles (SEPs) which are produced by explosive dissipation events (e.g., flares) in the sun's corona [Mason et al., 1994] and which can represent intense heavy ion-rich particle enhancements at Earth. Also of interest are energetic storm particle (ESP) events which are due to strong acceleration caused by traveling interplanetary shock waves moving outward from the sun. These ESP events can represent the most intense high-energy ion events that are observed near Earth (e.g., Zwickl and Kunches [1989]).

As seen in Figure 1, there can also be strong acceleration of ions at the forward-reverse shock pairs that form typically beyond $1 \mathrm{AU}$ in association with corotating solar wind stream interactions regions [Gloeckler, 1984]. These "corotating ion" events can be frequent and regular sources of highenergy ions as the solar wind streams emerging from solar coronal holes propagate outward through the heliosphere. These corotating events often exhibit $\sim 27$-day periodicity if the solar wind streams are long-lived (as they often are during sunspot minimum conditions).

Another shock-related source of relatively energetic ions is the diffuse upstream ions accelerated upstream of the Earth's 


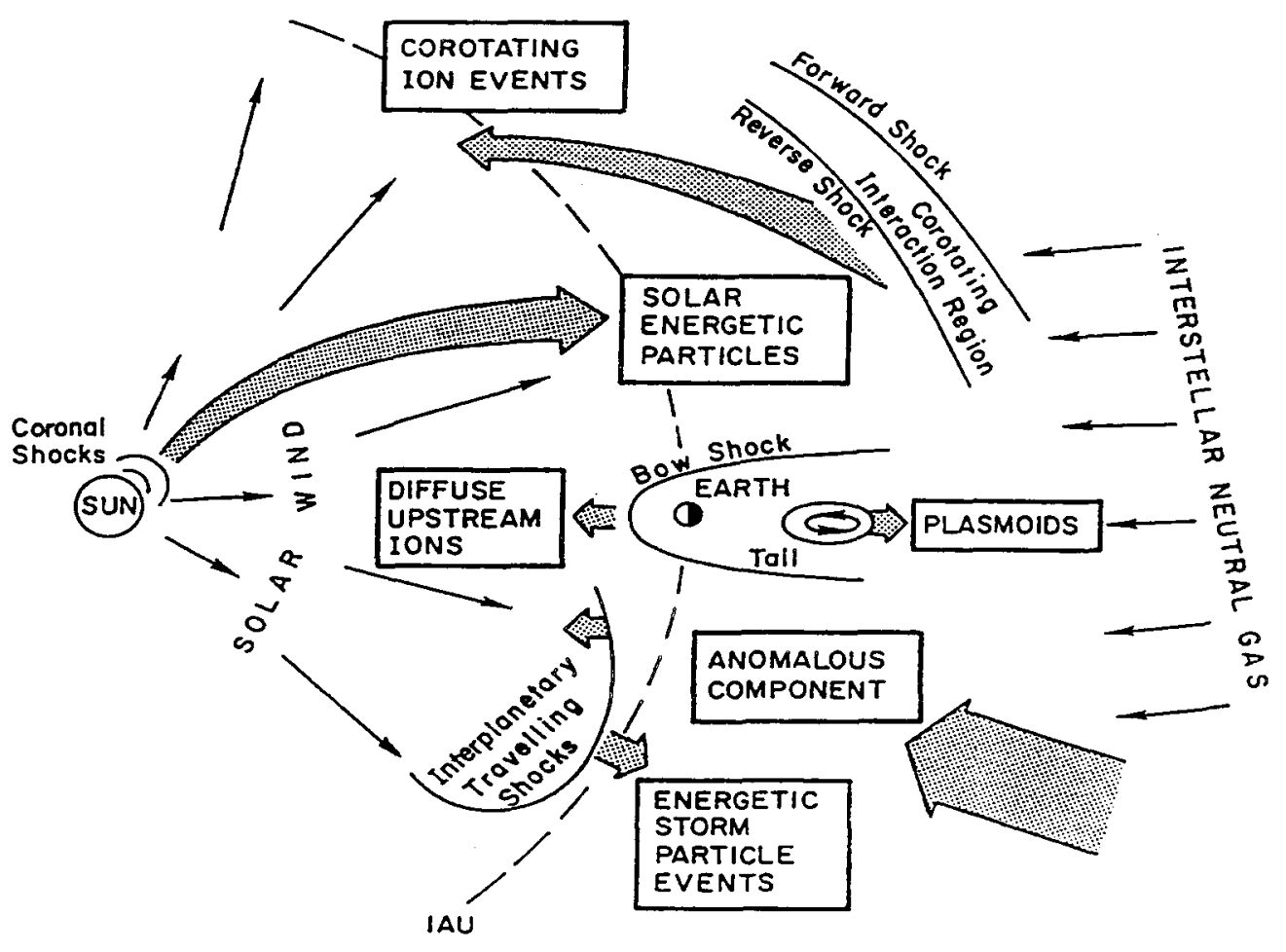

Figure 1. A schematic diagram illustrating the many types of energetic particle populations and associated acceleration mechanisms in the heliosphere (from Gloeckler [1984]).

bow shock [Scholer, 1985]. The present view is that solar wind ions initially "reflected" by the bow shock are further accelerated by Fermi processes in the quasi-parallel region of the bow shock. Solar protons and alpha particles may be accelerated to energies $>1 \mathrm{MeV}$ as they scatter back and forth between magnetic irregularities in the foreshock region upstream of the Earth.

A final population of interest is the anomalous cosmic ray component. The Solar, Anomalous, and Magnetospheric Particle Explorer (SAMPEX) spacecraft has detected a radiation belt surrounding the Earth that traps material from the nearby interstellar medium. This recently discovered belt is embedded in the lower of the two previously-known Van Allen belts. SAMPEX pinpointed the new belt whose existence was first predicted nearly 20 years ago [Blake and Friesen, 1977].

The new belt consists of trapped heavy ions, including the nuclei of atoms of nitrogen, oxygen, and neon which are part of the ACR (anomalous cosmic ray) component [Cummings et al., 1993]. These gases make up the tenuous $\left(\lesssim 1 \mathrm{~cm}^{-3}\right)$ interstellar gas which, if electrically neutral, can penetrate the heliosphere. Some of these neutral interstellar atoms are singly ionized by solar UV radiation, and are then accelerated to cosmic ray energies at the solar wind "termination shock". If one of these singly-charged cosmic rays encounters the Earth's atmosphere and loses its remaining electrons, it may become trapped in the Earth's magnetic field. Once inside the new belt, these atoms may be trapped for an extended period before leaking out into space or into the atmosphere. At present, the intensity of ACR oxygen inside the belt is about 400 times greater than in interplanetary space. The rate of trapping varies with the interplanetary ACR intensity, and as a result, the intensity of ions trapped in the belt varies by perhaps a factor of 100 to 1000 over the solar cycle.

As noted by Scholer [1979], any high energy particles which impinge upon the magnetosphere can, with finite probability, penetrate the magnetopause. Thus, it is likely that the magnetosphere is populated to some extent by all of the above sources. The relevant question is not whether external sources populate the radiation belts, but rather the question is whether a given source is significant-or even dominant-at a particular time in a given energy range.

\section{JOVIAN ELECTRONS AS A POSSIBLE RADIATION BELT SOURCE}

Electrons from Jupiter were first recognized by Teegarden et al. [1974] as the source of "quiet-time" cosmic ray electron increases. Various groups have, since the first suggestion of Teegarden et al., reported on the source characteristics, the interplanetary propagation, and the observed properties at $1 \mathrm{AU}$ of Jovian electrons outside the terrestrial magnetosphere (e.g., Krimigis et al. [1975]; Mewaldt et al. [1976]; Chenette et al. [1977]). 
BAKER ET AL. 51

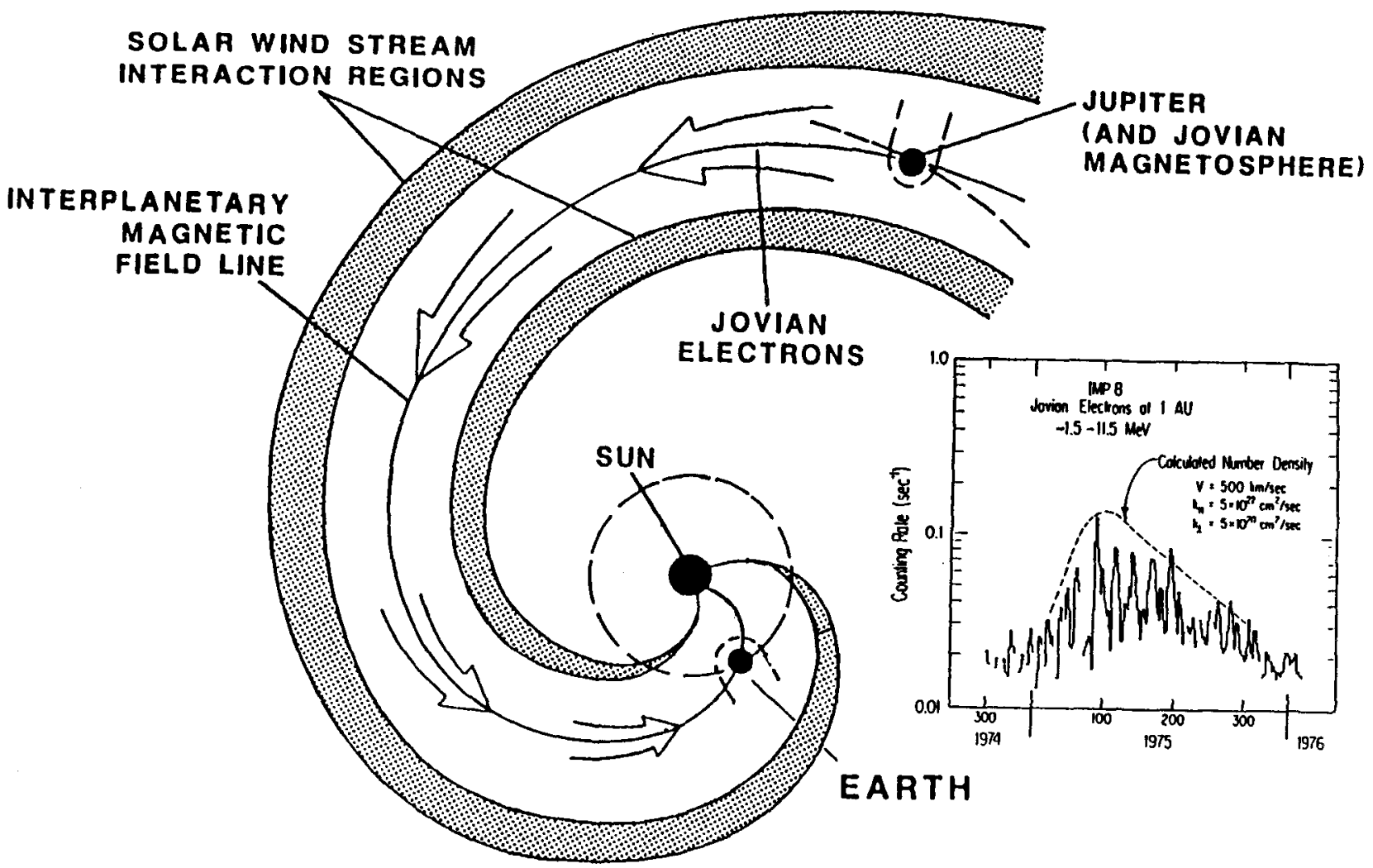

Figure 2. Diagram viewing downward from the north pole of the sun showing solar wind stream interaction regions and the possible path followed by Jovian electrons along interplanetary magnetic field lines toward Earth. Inset: 48-hr averages of IMP-8 $1.5-11.5 \mathrm{MeV}$ electron counting rates for late 1974 to early 1976 . The dashed curve is the predicted shape of peak Jovian electron intensities in the convection-diffusion model (after Conlon [1978]).

Jovian electrons $(E \lesssim 10 \mathrm{MeV})$ both at Jupiter and in the interplanetary medium near Earth have a spectrum with a power law spectral index $\gamma<2$ [Baker and Van Allen, 1976; Mewaldt et al., 1976]; which is remarkably hard; in fact this spectral feature has been used by most experimenters to identify Jovian electrons and to separate out terrestrial and solar backgrounds from the Jovian signal [Krimigis et al., 1975; Mewaldt et al., 1976; Chenette et al., 1977]. By way of contrast, in most regions of the terrestrial magnetosphere (as at 6.6 $R_{\mathrm{E}}$ ) and in solar energetic particle events, electron spectra have $\gamma \gtrsim 3$.

Also, Jovian electrons tend to consist of flux increases of several days duration which then recur with 27-day periodicities (e.g., Teegarden et al. [1974]; Mewaldt et al. [1976]). Furthermore, these electrons appear after high speed solar wind streams have gone past the Earth and, therefore, Jovian electrons appear at $1 \mathrm{AU}$ during periods of declining solar wind speed and low $K_{\mathrm{p}}$. The 27-day periodicity of the Jovian increases has been attributed by Conlon [1978] to the effects of recurrent, fast solar wind streams overtaking slower plasma which form corotating interaction regions (CIRs). The CIRs then form barriers to the cross-field diffusion of Jovian electrons, and thus the Jovian particles are constrained to propag- ate within the region between CIRs. The entire heliospheric CIR pattern rotates about the sun with the 27-day solar rotation period; when Jupiter and Earth are both within a given CIR "cavity", a Jovian increase is seen at Earth (see Figure 2).

A third point is that Jovian electron increases at $1 \mathrm{AU}$ exhibit a long-term periodicity of $\sim 13$ months which is the synodic Jovian period as viewed from the Earth [Chenette et al., 1977; Teegarden et al., 1974]. The modulation arises from the fact that once every 13 months Earth and Jupiter are directly connected along the ideal average IMF spiral field line and fluxes will be high during this period; low fluxes result when Earth is far from nominal connection since Jovian electrons must then diffuse large distances across interplanetary field lines to reach the Earth.

In Figure 3 we show a portion of CPA (Charged-Particle Analyzer) $1.4-2.0 \mathrm{MeV}$ electron data at geostationary orbit for 1977 -78. These data are from Baker et al. [1979] showing magnetospheric trapped particles. In eight of nine months during this period, Baker et al. identified the time of peak Jovian electron flux increases outside the magnetosphere (from Caltech IMP-8 data). Each of these peak Jovian electron times is shown by the dot in Figure 3. The missing dot (September 1977) corresponds to a time of a large solar 


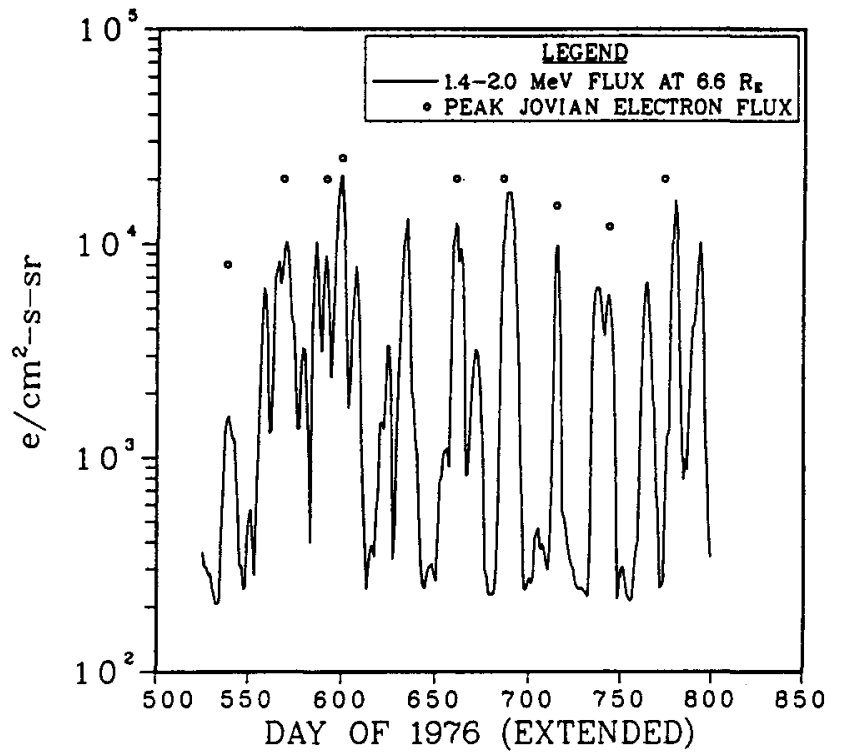

Figure 3. Charged-particle analyzer electron data (1.4-2.0 MeV) for 1977-78 at geostationary orbit. The dots show times that Jovian electron fluxes peaked outside the Earth's magnetosphere (from Baker et al. [1979]).

flare electron increase.

It is seen by Figure 3 that Jovian electron increases occur, in close temporal association with most of the major highenergy flux peaks at $6.6 R_{\mathrm{E}}$. When examined on a more detailed basis, it is observed that the Jovian electrons outside the magnetosphere commonly peak $1-3$ days prior to the peak seen in electrons at $6.6 R_{\mathrm{E}}$ inside the magnetosphere. As noted, Jovian electrons have very hard energy spectra $(\gamma \leq 2)$ by magnetospheric standards and similar spectral hardening occurs during each of the flux peaks in Figure 3 as well.

Based on results such as those shown in Figure 3, Baker et al. [1979] sketched an external source scenario. In this model Jovian electrons, controlled by interplanetary solar wind stream structure, appear regularly in the vicinity of Earth. Such Jovian electrons are observed down to energies as low as $\sim 200 \mathrm{keV}$ and they can readily enter the distant plasma sheet where the magnetic field is weak. The Jovian population (distinct because of its very hard spectrum) then becomes part of the plasma sheet population and begins to participate in the overall magnetospheric dynamics. During sunward convection in the plasma sheet, Jovian electrons are moved nearer the Earth; during substorms the Jovian population is convected strongly with plasma sheet particles and is "injected" into the outer radiation region. Inward radial diffusion would also be significant for such particles.

A question addressed by Baker et al. [1979] is whether Jovian electron absolute intensities at a given energy outside the magnetosphere are similar to those at geostationary orbit. It was found that $>1 \mathrm{MeV}$ fluxes at geostationary orbit are of the order of $10^{\widetilde{3}}$ times higher than in the interplanetary medium. However, the first adiabatic invariant $\left(\mu \sim E^{2} / \mathrm{B}\right)$ should be conserved [Schulz and Lanzerotti, 1974] as elec-
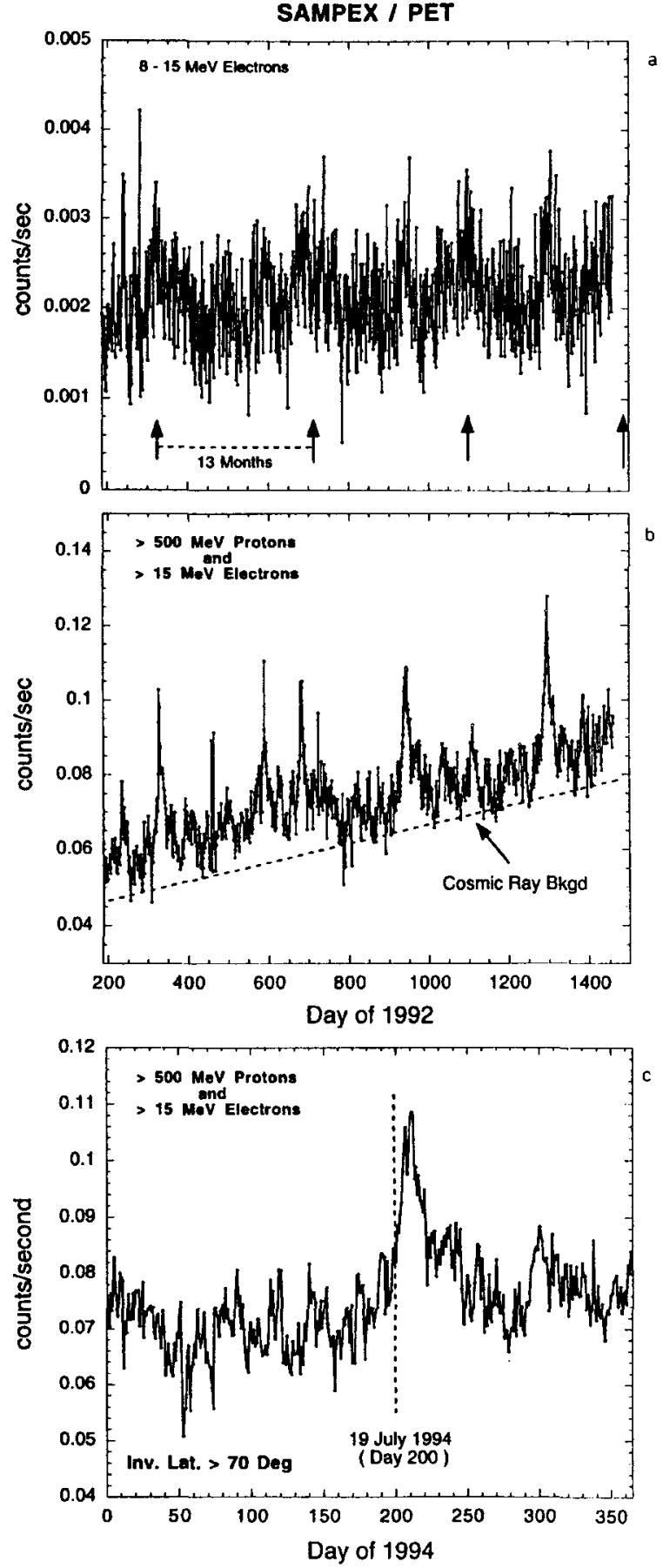

Figure 4. High energy electron fluxes measured by the SAMPEX spacecraft over the polar caps. (a) This figure shows daily averages of 8-15 MeV electrons from July 1992 through 1995 and suggests a 13-month modulation. (b) This figure shows higher energy data that include galactic protons plus $>15 \mathrm{MeV}$ electrons. (c) This figure shows a detail of Figure $4 b$ for 1994. A large flux increase on Day 200 (19 July) of 1994 may have been caused by Comet Shoemaker-Levy 9 impact at Jupiter. 
trons are transported from interplanetary space (or deep in the tail) to $6.6 R_{\mathrm{E}}$. Thus, the ambient magnetic field will be increased from $\sim 5 \mathrm{nT}$ to over $100 \mathrm{nT}$. This factor of $\sim 20$ increase in $B$ and conservation of $\mu$ (plus the hard spectrum) can account for higher fluxes at geostationary orbit by a factor of about 20. Also, if the characteristic source time is short compared to the characteristic loss time at $6.6 R_{\mathrm{E}}$, then Jovian fluxes could build up further in the outer zone. Source time scales should be associated primarily with substorm occurrence frequency. Loss time scales should be associated with radial diffusion times and, also, lifetimes against pitch angle scattering into the loss cone. Since several substorms occur, on average, each day, it is not unreasonable to suppose that a buildup could occur. Furthermore, Jovian electrons appear for several days outside the magnetosphere and 10-20 substorms can occur in this period, thus causing a buildup of fluxes in the outer zone.

Based on early work such as described above, it seemed possible that Jovian electrons would play an important role in populating the electron radiation belts. Subsequent work [Christon et al., 1989] suggested that Jovian electrons are not the dominant cause of low-to-moderate energy (0.2$5.0 \mathrm{MeV}$ ) electron enhancements at geostationary orbit. Recent SAMPEX data [Baker et al., 1994] also suggests that internal magnetospheric acceleration is the dominant source of typical relativistic electron enhancements in the outer radiation zone. However, it may still be the case that the highest energy trapped electrons are introduced by the Jovian source [Baker et al., 1986].

An interesting illustration of very high-energy electron influence from an external source is shown in Figure 4. Figure 4a shows measurements over the period 1992-95 from the P4-P7 sensors of the PET telescope of SAMPEX (Looper et $a l$. [1994] and references therein). The data are daily averages for times when SAMPEX was at invariant latitudes above $70^{\circ}$. These data correspond, therefore, to polar cap fluxes and are a measure of $\sim 8-15 \mathrm{MeV}$ electrons essentially in the interplanetary medium. Although statistical fluctuations are large, the data suggest both by the highest maximum count rates and by the trends in the minimum count rates that there is a systematic modulation of the fluxes with something over a 1-year period. We show by the vertical arrows periods separated by 13 months. This 13 -month period looks very consistent with the modulation seen in Figure $4 \mathrm{a}$ and supports the view that Jovian electrons are seen by SAMPEX over the polar caps.

An even higher PET energy range for electrons (which also includes galactic cosmic ray protons $>500 \mathrm{MeV}$ ) is shown in Figure $4 \mathrm{~b}$. The period covered is the same as Figure $4 \mathrm{a}$ and is again for latitudes $>70^{\circ}$. The solar cycle trend in the background level (indicated by the dashed line) is consistent with galactic cosmic ray modulation. Of interest are the long-lasting events extending well above the background level (for example, around Day 325, Day 590, Day 925, and Day 1275). Other SAMPEX data suggest that these are not proton enhancements and thus they are most likely very energetic electron events. During the period 16-22 July 1994, fragments of Comet Shoemaker-Levy 9 plunged into the upper atmosphere of Jupiter. Numerous scientific reports have described the visible, IR, and UV wavelength observations of these huge impacts. Recent papers have also discussed extreme ultraviolet, $\mathrm{X}$-ray, and radio signatures detected at
Earth in association with the S/L 9 impacts (e.g., dePater et al. [1995]; Waite et al. [1995]). The radio emissions, in particular, suggest that magnetospheric electrons at Jupiter in the energy range $1-300 \mathrm{MeV}$ were significantly perturbed by the comet fragments [dePater et al., 1995].

In Figure $4 c$ we expand a portion of the SAMPEX record and we examine the high energy interplanetary electron flux variations. We find evidence that energetic electrons increased substantially in flux beginning about 19 July 1994 ( $\sim$ Day 200). The clearest increase was for $>5 \mathrm{MeV}$ electrons which remained elevated in flux for $\sim 2$ months. The spectrum of these electrons was apparently quite hard since, as seen in Figure $4 c$, there was a very clear flux enhancement in electrons with $E>15 \mathrm{MeV}$. This is consistent with the hard energy spectrum seen previously for Jovian electrons. Jupiter and Earth were not ideally connected via the nominal interplanetary magnetic field in July 1994 . Therefore, an even stronger signal might have been seen if ideal magnetic connection had obtained.

We continue to examine high-energy electron data with SAMPEX and other magnetospheric spacecraft in order to understand the acceleration and transport processes that ultimately populate the highly variable outer zone. The large peak in July 1994 is suggestive of a S/L 9 effect, but the question remains as to what caused the other large peaks in Figure $4 b$.

\section{SOLAR ENERGETIC ELECTRON SOURCE}

As noted above, it has been well established that energetic protons and other ions from the sun can penetrate into the terrestrial magnetosphere [Scholer, 1979; Fennell, 1973]. Some portion of these ions can be trapped and can constitute a reasonably persistent (days to weeks) component of the magnetospheric particle environment. A question arises as to whether or not solar energetic electrons are also a significant source of outer zone electron flux enhancements.

In an earlier study, Baker et al. [1986] examined data from IMP-8 and other available upstream spacecraft and compared energetic electron measurements with concurrent data of similar energy at geostationary orbit. It was concluded that for electrons with $E \gtrsim 0.1 \mathrm{MeV}$, there was not a one-to-one relationship between solar electron events and geostationary electron enhancements. In fact, in most cases there were no large solar electron events that could be associated with geostationary recurrent flux events.

New measurements from SAMPEX allow a further examination of this question of solar electron entry. In Figure 5a, we show SAMPEX data from January-February 1994. The measurements are for the northern polar cap region with a selection criterion applied such that magnetic latitude is greater than $70^{\circ}$. In the open magnetosphere model, this criterion would suggest that at such high latitudes SAMPEX was sampling essentially interplanetary-connected field lines. Thus, solar particles would have rather direct access to this region (e.g., Fennell [1973]).

A large solar energetic particle event was observed on 2026 February 1994 (see Baker et al. [1995]). As seen in Figure $5 \mathrm{a}$, the $2-6 \mathrm{MeV}$ electron flux measured by the ProtonElectron Telescope (PET) onboard SAMPEX rose rapidly above background levels on $\sim 21$ February and intensities 


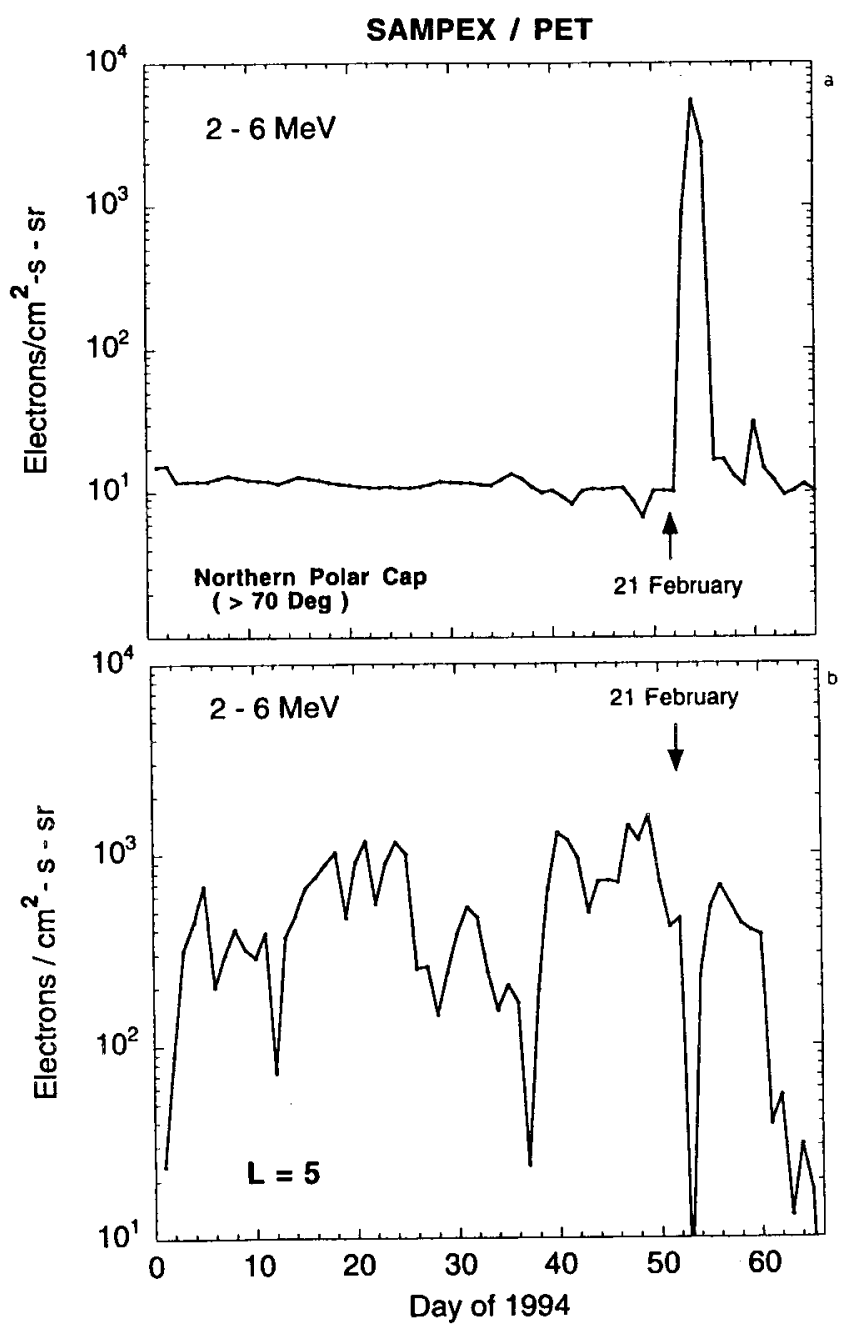

Figure 5. (a) Polar cap electron fluxes (2-6 MeV) measured by SAMPEX during Jan.-Feb. 1994. A solar energetic electron event commenced on 21 February 1994. (b) Same as (a) but showing trapped electron fluxes measured at lower latitudes for $L=5$. Electron intensities decrease rapidly on 21 February.

remained high for several days. These data suggest a strong solar electron event in this case.

In Figure 5b, we show measurements of electron fluxes again in the 2-6 MeV channel of SAMPEX for the same period of time as in Figure 5a. However, in Figure 5b we show data for the trapping region at $L=5$. In January and early in February the figure shows that there were several large increases (and decreases) in the relativistic electron flux in the outer zone. Previous papers [e.g., Baker et al. [1994]) showed that these electron enhancements were driven by high speed solar wind streams hitting the magnetosphere. However, on 21 February-when the polar cap fluxes were increasing dramatically-the $2-6 \mathrm{MeV}$ electron flux at $L=5$ actually dropped precipitously. Thus, despite there being strong and rapid access of solar electrons to the polar cap
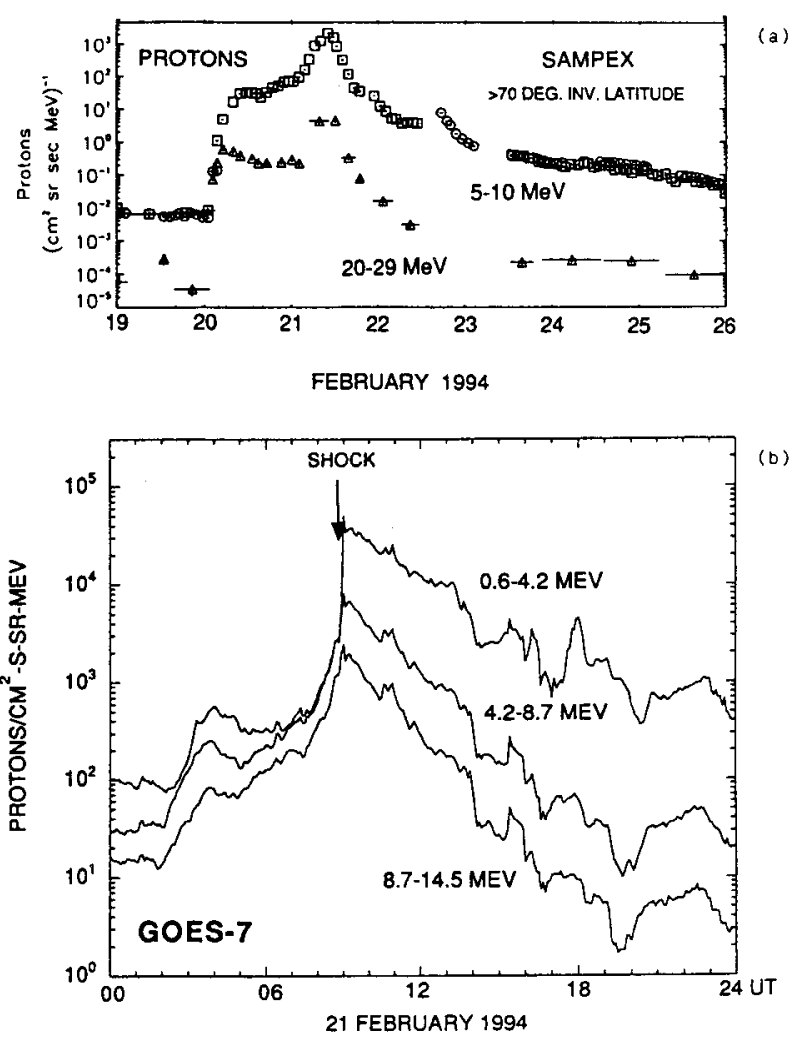

Figure 6. (a) Summary of proton fluxes for the period 19-25 February 1994 as measured over the polar caps by SAMPEX sensors. (b) Data from GOES-7 at geostationary orbit for the period 0000-2400 UT on 21 February 1994. (From Baker et al. [1995]).

in this case, such electrons did not (at least not immediately) enter into the outer zone trapping region. Thus, it appears that solar energetic electrons do not constitute a prompt or dominant source of outer zone electrons. By the same token, during all of January and early February 1994, it appears from Figure 5 that there were numerous large changes in the outer zone $(L=5)$ electron fluxes (Day 14-24 and Day 38-49) that had no counterpart in the polar cap flux. These results suggest that at these times neither solar nor Jovian electrons were the dominant cause of magnetospheric trapped electron events.

In some contrast we see clear evidence that, for the February 1994 event, solar protons had rather ready access to the outer portion of the Earth's radiation belt. In Figure $6 a$ we show high latitude proton measurements from SAMPEX in the energy ranges $5-10 \mathrm{MeV}$ and $20-29 \mathrm{MeV}$. The period shown is 19-25 February. As described by Baker et al. [1995], the proton flux seen by SAMPEX above magnetic latitude $70^{\circ}$ jumped up early on 20 February and then a further large increase occurred on 21 February when a strong shock wave struck the magnetosphere. Figure $6 \mathrm{~b}$ shows data from the GOES-7 spacecraft at geostationary orbit for 21 February. Energetic proton fluxes were elevated on 20 February at GOES-7 [R. Zwickl, private communication], but the flux 
at geostationary orbit increased very sharply and substantially at $\sim 0900 \mathrm{UT}$ as the interplanetary shock wave passed through the magnetosphere [Baker et al., 1995]. Thus, both SAMPEX and GOES-7 saw similar proton signatures at about the same time. Hence, solar and interplanetary ions can constitute a prompt outer magnetosphere contribution, but solar electrons seem not to be such a strong source.

\section{SUMMARY}

Long-term data show a high coherence of relativistic electron flux variations throughout the entire outer radiation zone (Baker et al. [1994] and references therein). It is seen that strong high-energy electron modulation occurs on 27-day time scales. Some influence of Jovian electrons is possible (especially at the highest energies). There is little evidence for a direct solar energetic electron source within the radiation belts. Rather, there is ample evidence that outer zone relativistic electrons are acclerated within the magnetosphere on relatively short time scales $(\lesssim 1$ day). Such acceleration is driven by the impact of high-speed solar wind streams on the magnetosphere. On the other hand, there is a direct evidence that solar energetic ions can penetrate deeply into the Earth's outer magnetospheric regions, thus allowing flare and shock-generated ions to be present for many days after solar ion events commence.

Acknowledgements. This work was supported by NASA grants through the SAMPEX program. We thank X. Li for useful discussions.

\section{REFERENCES}

Baker, D.N. and J.A. Van Allen, Energetic electrons in the Jovian magnetosphere, J. Geophys. Res., 81, 617, 1976.

Baker, D.N., P.R. Higbie, R.D. Belian and E.W. Hones, Jr., Do Jovian electrons influence the terrestrial outer radiation zone? Geophys. Res. Lett., 6, 531, 1979.

Baker, D.N., J.B. Blake, R.W. Klebesadel and P.R. Higbie, Highly relativistic electrons in the Earth's outer magnetosphere, I. Lifetimes and temporal history 1979-1984, J. Geophys. Res., 91, $4265,1986$.

Baker, D.N., J.B. Blake, S. Kanekal, B. Klecker and G. Rostoker, Satellite anomalies linked to electron increase in the magnetosphere, EOS, 75, 401, 1994.

Baker, D.N., S. Kanekal, J.B. Blake and J.H. Adams, Jr., ChargedParticle Telescope on Clementine, J. Spacecraft Rockets, 32, $1060,1995$.

Blake, J.B. and L.M. Friesen, A technique to determine the charge state of the anomalous low energy cosmic rays, 15 Int. Cosm. Ray Conf., 2, 341, 1977.

Chenette, D.L., T.F. Conlon, K.R. Pyle and J.A. Simpson, Observations of Jovian electrons at $1 \mathrm{AU}$ throughout the 13-month Jovian synodic year, Ap. J. (Letters), 215, L 95, 1977.

Christon, S.P., D.L. Chenette, D.N. Baker and D. Moses, Relativistic electrons at geosynchronous orbit, interplanetary electron flux, and the 13-month Jovian synodic year, Geophys. Res. Lett., 16, $1129,1989$.
Conlon, T.F., The interplanetary modulation and transport of Jovian electrons, J. Geophys. Res., 83, 541, 1978.

Cowley, S.W.H., Plasma populations in the simple open model magnetosphere, Space Sci. Rev., 26, 217, 1980.

Cummings, J.R., A.C. Cummings, R.A. Mewaldt, R.S. Selesnick, E.C. Stone and T.T. vonRosenvinge, New evidence for anomalous cosmic rays trapped in the magnetosphere, Geophys. Res. Lett., 20, 2003-2006, 1993.

dePater, I., et al., Outburst of Jupiter's synchrotron radiation after the impact of comet Shoemaker-Levy 9, Science, 268, 1879, 1995.

Fennell, J.F., Access of solar protons to the Earth's polar cap, $J$. Geophys. Res., 78, 1036, 1973.

Fisk, L.A., Increases in the low-energy cosmic ray intensities at the front of propagating interplanetary shock waves, J. Geophys. Res., 76, 1662, 1971.

Gloeckler, G., Characteristics of Solar and Heliospheric Ion Populations Observed Near Earth, Adv. Space Res., 4, 127-137, 1984.

Gloeckler, G. and D.C. Hamilton, AMPTE ion composition results, Phys. Scr., T17, 73, 1987.

Krimigis, S.M., E.T. Sarris and T.P. Armstrong, Observations of Jovian electron events in the vicinity of Earth, Geophys. Res. Lett., 2, 561, 1975.

Looper, M.D., J.B. Blake, R.A. Mewaldt, J.R. Cummings and D.N. Baker, Observations of the remnants of the ultrarelativistic electrons injected by the strong SSC of 24 March 1991, Geophys. Res. Lett., 21, $2079,1994$.

Mason, G.M., J.E. Mazur and D.C. Hamilton, Heavy ion isotopic anomalies in $3 \mathrm{He}$-rich solar particle events, Astrophys. J., 425 , 843-848, 1994.

Mewaldt, R.A., E.C. Stone and R.E. Vogt, Observations of Jovian electrons at $1 \mathrm{AU}$, J. Geophys. Res., 81, 2397, 1976.

Scholer, M., Energetic solar particle behavior in the magnetosphere, in Solar-Terrestrial Predictions Proceedings, 2, 446, edited by R.F. Donnelly, NOAA, Boulder, CO, 1979.

Scholer, M., Diffuse acceleration, in Collisionless Shocks in the Heliosphere: Reviews of Current Research, Geophys. Monogr. Ser., 35, edited by B.T. Tsurutani and R.G. Stone, pp. 287-301, AGU, Washington, DC, 1985.

Schulz, M. and L.J. Lanzerotti, Particle Diffusion in the Radiation Belts, Springer, New York, 1974.

Teegarden, B.J., F.B. McDonald, J.H. Trainor, W.R. Webber and E.C. Roelof, Interplanetary MeV electrons of Jovian origin, $J$. Geophys. Res., 79, 3615, 1974.

Waite, J.H., Jr., et al., ROSAT observations of x-ray emissions from Jupiter during the impact of Comet Shoemaker-Levy 9, Science, $268,1598,1995$.

Zwickl, R.D. and J. Kunches, EOS, 70, 1258, 1989 (abstract).

D.N. Baker, LASP, University of Colorado, Campus Box 392, Boulder, CO 80309-0392

S.G. Kanekal, NASA/Goddard Space Flight Center, Greenbelt, MD, USA

M.D. Looper and J.B. Blake, Aerospace Corp., Los Angeles, CA, USA

R.A. Mewaldt, California Inst. of Tech., Pasadena, CA, USA 